\title{
Urinary continence outcomes of four years of follow-up and predictors of early and late urinary continence in patients undergoing robot-assisted radical prostatectomy
}

\author{
Xing Li ${ }^{1}$, Huan Zhang ${ }^{1}$, Zhuo Jia ${ }^{1}$, Yunpeng Wang ${ }^{1}$, Yong Song ${ }^{1}$, Limin Liao ${ }^{2^{*}}$ and Xu Zhang ${ }^{1 *}$
}

\begin{abstract}
Background: The robot-assisted radical prostatectomy (RARP) has been widely applied in recent years; however, only a few studies are reported about long-term urinary continence after surgery. The present study aimed to examine the outcomes of continence rates (CRs) and determine the risk and protective factors of urinary continence in patients with prostate cancer (PCa) undergoing RARP.

Methods: This retrospective study included 650 patients treated with RARP with perioperative data and at least one year of follow-up from September 2009 to November 2017. Also, the preoperative, intraoperative, and postoperative parameters of the patients were analyzed. Continence was defined as no pad use. Early and late continence was defined as the return of urinary continence within 3 months and beyond 12 months post-surgery, respectively. CRs were examined from 1 to 48 months postoperatively. Logistic regression analysis evaluated the association between the predictive factors and urinary continence in the early and late stages.

Results: No significant difference was detected in the CR from 12 to 48 months postoperatively $(P=0.766)$. Logistic regression analysis proved that pelvic lymph node dissection (PLND) was a significant risk factor of urinary continence at 1 month. Nerve-sparing (NS) was a significant protective factor of urinary continence at 1, 3, and 6 months. Advanced age was an independent risk factor of urinary continence at 6, 12, and 24 months. Other variables were not statistically significant predictors of urinary continence.

(Continued on next page)
\end{abstract}

\footnotetext{
* Correspondence: Imliao@263.net; xzhang@foxmail.com

Department of Urology, Rehabilitation School of Capital Medical University,

China Rehabilitation Research Center, No. 10 Jiaomen North Road, Fengtai

District, Beijing 100068, China

'Department of Urology, Medical College of People's Liberation Army,

Chinese People's Liberation Army General Hospital, No. 28 Fuxing Road,

Haidian District, Beijing 100853, China
}

(c) The Author(s). 2020 Open Access This article is licensed under a Creative Commons Attribution 4.0 International License, which permits use, sharing, adaptation, distribution and reproduction in any medium or format, as long as you give appropriate credit to the original author(s) and the source, provide a link to the Creative Commons licence, and indicate if changes were made. The images or other third party material in this article are included in the article's Creative Commons licence, unless indicated otherwise in a credit line to the material. If material is not included in the article's Creative Commons licence and your intended use is not permitted by statutory regulation or exceeds the permitted use, you will need to obtain permission directly from the copyright holder. To view a copy of this licence, visit http://creativecommons.org/licenses/by/4.0/ The Creative Commons Public Domain Dedication waiver (http://creativecommons.org/publicdomain/zero/1.0/) applies to the data made available in this article, unless otherwise stated in a credit line to the data. 
(Continued from previous page)

Conclusions: The current results demonstrated that CR gradually improved with time within 1 year and stabilized 1 year after the surgery. PLND, NS, and age were significant determinants of continence in the early and late stages, respectively. These parameters could be used for preoperative identification of patients at high risk and counseling about postoperative expectations for urinary continence.

Keywords: Prostate cancer, Prostatectomy, Robotics, Urinary incontinence, Continence

\section{Background}

Despite advances in surgical technique and methodology, postprostatectomy urinary incontinence (UI) remains a significant adverse event that leads to decreased quality of life $[1,2]$. Several factors are involved in the recovery of urinary continence after radical prostatectomy (RP) [3, 4]. Since patients have learned about this disease from the media, it is necessary to predict the recovery of urinary continence early in order to minimize the patients' concerns and embarrassment [5].

The technical progress has facilitated the increasing use of robot-assisted radical prostatectomy (RARP) worldwide [6]. Because the procedure can induce different degrees of damage to the bladder and urethra, UI is inevitable despite the assistance of robotic systems $[7,8]$. This in turn, places physical and psychosocial burden on patients.

Reportedly, urinary continence improves only slightly in the 12 months after the surgery [2]. Hence, previous reports mainly addressed continence rates (CRs) and potential predictors within 1 year post-RARP. However, continence may continue to develop at 2 years postsurgery [7, 9, 10]. Limited data are available for longer follow-up with urinary continence after 24-48 months. In addition, a series of studies reported outcomes at 1 year after surgery, and most of them included a small number of patients $(<200)$. Moreover, the follow-up period was discontinuous or incomplete. For example, Yanagiuchi et al. examined and identified the outcomes at 1 and 3 months post-RARP, while Olgin et al. and Haga et al. analyzed and evaluated the outcomes at 3 and 12 months after the surgery $[8,11,12]$. In addition, Honda et al. reported continuous outcomes from 1 to 6 months postoperatively [13].

To the best of our knowledge, continuous follow-up data from 1 to 48 months on CRs and predictive factors for urinary continence after RARP with a high number of patients have not been collected. Therefore, in this retrospective study, we examined the outcomes of CRs at 1, 3, 6, 12, 24,36 , and 48 months after the surgery and determined the risk and protective factors of urinary continence.

\section{Methods}

Patients diagnosed with clinically localized prostate cancer and received treatment from September 2009 to
November 2017 at our institution were studied in a retrospective manner. Only patients who completed the outpatient visits or telephonic interviews for at least one year were enrolled. Patients with incomplete data and those unavailable for follow-up of continence were excluded from the study. Patients who underwent preoperative transurethral resection, enucleation of the prostate, radioactive seed implantation, orchidectomy, bladder neck, urethral, or pelvic surgery, and retropubic radical prostatectomy (RRP) or laparoscopic radical prostatectomy (LRP) were also excluded. Finally, a total of 650 eligible patients were analyzed. The schematic is illustrated in Fig. 1.

Data were collected from cases performed by three surgeons. RARP was performed by a transperitoneal approach. Nerve-sparing (NS) procedures were attempted in all potent patients at a clinical stage of $\mathrm{T} 1$ or $\mathrm{T} 2$, prostate-specific antigen (PSA) $\leq 10 \mathrm{ng} / \mathrm{mL}$, and Gleason score $\leq 7$. To preserve the urethral length, it was performed proximally close to the prostate while dissecting the urethra. Pelvic lymph node dissection (PLND) was performed selectively to collect samples in intermediateand high-risk patients [14]. Urethrovesical anastomosis was carried out using 2-0 monofilament sutures with a $5 / 8$ needle. Typically, a catheter was placed and removed at 3 weeks after the surgery.

Clinical data on the demographic characteristics and surgery-related variables were retrieved from the patient's medical records. Together with the follow-up records, the data were assimilated into a database in this retrospective study. The factors assessed included age at the operation, body mass index (BMI), comorbidities such as hypertension (HP), diabetes mellitus (DM), coronary heart disease (CHD), and cerebrovascular diseases (CD), serum PSA level, prostate volume (PV), biopsy-determined Gleason score, clinical stage, operation time (OT), NS and PLND during surgery, and the duration of indwelling catheter (DIC) after the surgery. UI was defined as any leakage of urine after the surgery. Early and late continence was defined as the return of urinary continence within 3 months and beyond 12 months after surgery, respectively [12].

\section{Statistical analysis}

SPSS version 19.0 (SPSS, Inc., Chicago, IL, USA) was used for statistical analysis. Data are reported as mean \pm 


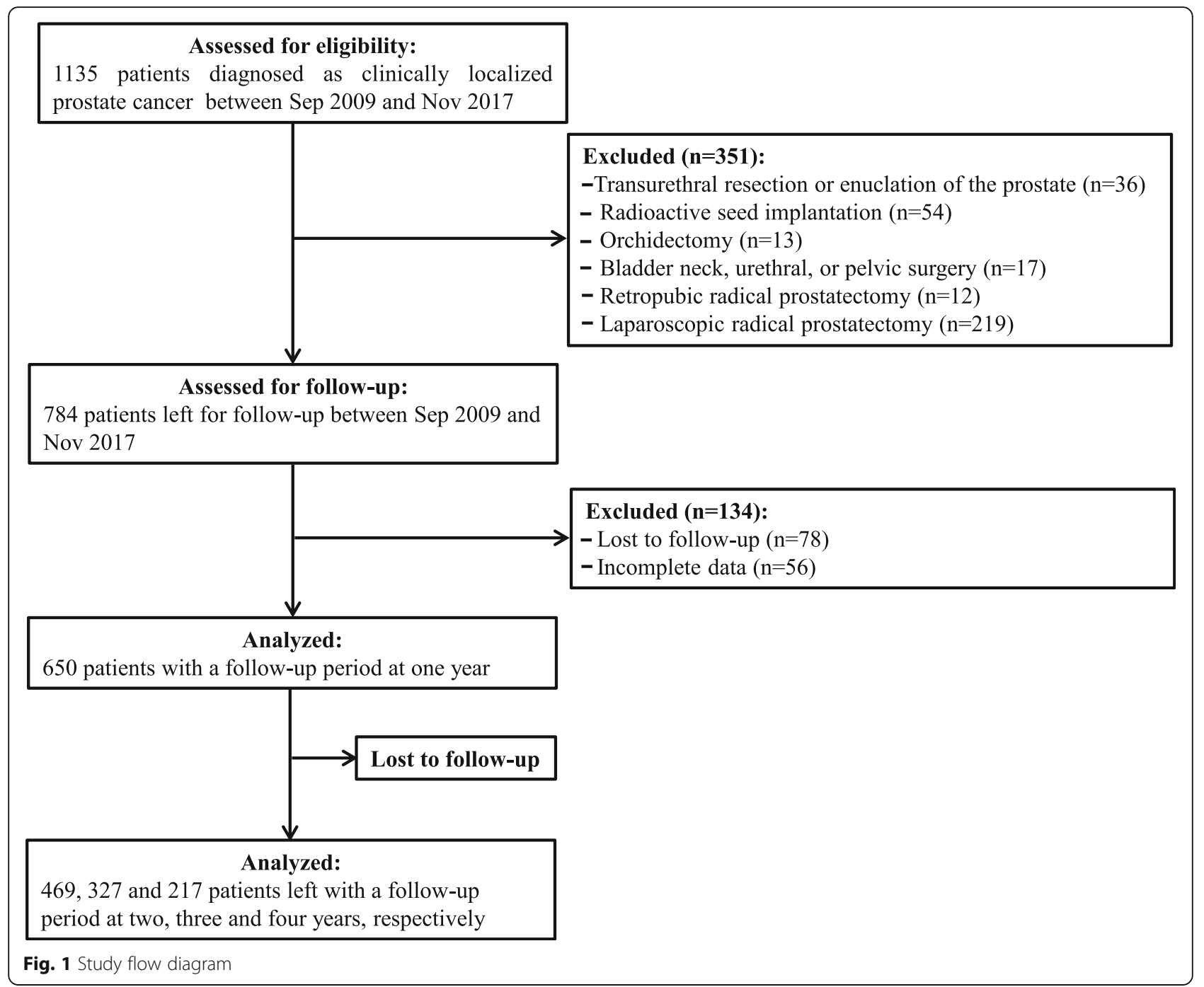

standard deviation or percentage. The chi-square test was used to compare the CRs at 12, 24, 36, and 48 months. The independent sample t-test or chi-square test was used to compare the predictive factors for urinary continence after RARP. All the above factors that might affect urinary continence were included in the univariate and multivariate logistic regression analysis to identify the risk and protective factors. $P<0.05$ was considered statistically significant.

\section{Results}

CRs at $1,3,6,12,24,36$, and 48 months post-surgery were $40.62,60.92,71.38,78.77,79.96,79.51$, and $76.50 \%$, respectively (Table 1), which improved gradually within one year. The comparison of CRs at 12 and 48 months did not reveal any significant differences in the continence outcomes during the 4-year follow-up $(P=0.766)$. Consequently, $\mathrm{CR}$ was found to be stabilized 1 year after the operation.
Furthermore, the demographic data and baseline clinical characteristics of the two groups (continence and incontinence groups) at 1 and 48 months were compared (Tables 2, 3, and 4). Fewer patients had NS, and a large number of patients had PLND in the incontinence group than those in the continence group during the surgery at a 1-month follow-up $(P<0.05)$ (Table 2$)$. Similarly, more

Table 1 Continence data from 1 to 48 months after RARP

\begin{tabular}{ll}
\hline 1 month $(N=650)$ & No. continence (\%) \\
3 months $(N=650)$ & $264(40.62 \%)$ \\
6 months $(N=650)$ & $396(60.92 \%)$ \\
12 months $(N=650)$ & $464(71.38 \%)$ \\
24 months $(N=469)$ & $512(78.77 \%)$ \\
36 months $(N=327)$ & $375(79.96 \%)$ \\
48 months $(N=217)$ & $260(79.51 \%)$ \\
\hline
\end{tabular}

Abbreviation: RARP Robot-assisted radical prostatectomy 
Table 2 Comparison of parameters between continence and incontinence patients at 1 and 3 months after operation

\begin{tabular}{|c|c|c|c|c|c|c|}
\hline \multirow[t]{2}{*}{ Variable } & \multicolumn{3}{|l|}{1 Month } & \multicolumn{3}{|l|}{3 Months } \\
\hline & Continence $(N=264)$ & Incontinence $(N=386)$ & $P$ value & Continence $(N=396)$ & Incontinence $(N=254)$ & $P$ value \\
\hline Age (years) & $65.60 \pm 7.04$ & $65.63 \pm 7.94$ & 0.959 & $65.20 \pm 7.32$ & $66.27 \pm 7.94$ & 0.080 \\
\hline BMI $\left(\mathrm{kg} / \mathrm{m}^{2}\right)$ & $24.85 \pm 2.75$ & $25.14 \pm 2.98$ & 0.209 & $25.01 \pm 2.82$ & $25.02 \pm 3.01$ & 0.964 \\
\hline \multicolumn{7}{|l|}{ Comorbidities, no (\%) } \\
\hline $\mathrm{HP}$ & 77 (29.17\%) & $141(36.53 \%)$ & 0.051 & $122(30.81 \%)$ & $96(37.80 \%)$ & 0.066 \\
\hline DM & $38(14.39 \%)$ & 77 (16.06\%) & 0.563 & $64(16.16 \%)$ & $36(14.17 \%)$ & 0.493 \\
\hline $\mathrm{CHD}$ & $20(7.58 \%)$ & $43(11.14 \%)$ & 0.131 & $38(9.60 \%)$ & 25 (9.84\%) & 0.917 \\
\hline$C D$ & $9(3.41 \%)$ & $11(2.85 \%)$ & 0.685 & $10(2.53 \%)$ & $10(3.94 \%)$ & 0.309 \\
\hline PSA (ng/mL) & $27.65 \pm 32.92$ & $32.11 \pm 36.08$ & 0.109 & $28.65 \pm 32.17$ & $32.86 \pm 38.64$ & 0.133 \\
\hline PV (mL) & $37.73 \pm 20.20$ & $35.80 \pm 18.58$ & 0.211 & $37.15 \pm 19.34$ & $35.70 \pm 19.15$ & 0.348 \\
\hline Gleason score, no (\%) & & & 0.475 & & & 0.654 \\
\hline$\leq 6$ & 79 (29.92\%) & $114(29.53 \%)$ & & 117 (29.55\%) & 76 (29.92\%) & \\
\hline 7 & 95 (35.98\%) & $160(41.45 \%)$ & & $151(38.13 \%)$ & $104(40.94 \%)$ & \\
\hline$\geq 8$ & 78 (29.55\%) & 97 (25.13\%) & & $113(28.54 \%)$ & 62 (24.41\%) & \\
\hline Unknown & $12(4.55 \%)$ & 15 (3.89\%) & & $15(3.78 \%)$ & $12(4.73 \%)$ & \\
\hline Clinical T stage, no (\%) & & & 0.962 & & & 0.550 \\
\hline $\mathrm{T} 1 \mathrm{a}, \mathrm{b}$ & $1(0.37 \%)$ & $1(0.26 \%)$ & & $1(0.25 \%)$ & $1(0.39 \%)$ & \\
\hline T1c & 46 (17.42\%) & 68 (17.62\%) & & 69 (17.42\%) & 45 (17.72\%) & \\
\hline $\mathrm{T} 2 \mathrm{a}, \mathrm{b}$ & $102(38.64 \%)$ & 139 (36.01\%) & & 157 (39.65\%) & 84 (33.07\%) & \\
\hline $\mathrm{T} 2 \mathrm{C}$ & $103(39.02 \%)$ & $155(40.16 \%)$ & & $149(37.63 \%)$ & 109 (42.91\%) & \\
\hline Т3а & $1(0.37 \%)$ & $2(0.51 \%)$ & & $1(0.25 \%)$ & $2(0.79 \%)$ & \\
\hline $\mathrm{T} 3 \mathrm{~b}$ & $11(4.17 \%)$ & $21(5.44 \%)$ & & 19 (4.80\%) & 13 (5.12\%) & \\
\hline OT (mins) & $151.61 \pm 42.16$ & $153.81 \pm 48.78$ & 0.551 & $151.02 \pm 41.76$ & $155.86 \pm 52.30$ & 0.192 \\
\hline NS, no (\%) & 73 (27.65\%) & 48 (12.44\%) & 0.000 & 87 (21.97\%) & 34 (13.39\%) & 0.045 \\
\hline PLND, no (\%) & $121(45.83 \%)$ & 217 (56.22\%) & 0.009 & 204 (51.52\%) & $134(52.76 \%)$ & 0.757 \\
\hline DIC (days) & $21.22 \pm 10.83$ & $21.02 \pm 10.09$ & 0.810 & $21.28 \pm 11.46$ & $20.84 \pm 8.46$ & 0.601 \\
\hline
\end{tabular}

Abbreviations: BMI Body mass index, CHD Coronary heart disease, $C D$ Cerebrovascular diseases, DM Diabetes mellitus, DIC Duration of indwelling catheter, HP Hypertension, NS Nerve-sparing, OT Operation time, PSA Prostate-specific antigen, PV Prostate volume, PLND Pelvic lymph node dissection

patients in the continence group had intraoperative NS than those in the incontinence group, as observed at the 3-month follow-up $(P<0.05)$ (Table 2). Data from the 6- and 12month follow-ups are presented in Table 3. At a 6-month follow-up, the continence group more commonly had a NS procedure performed $(P<0.05)$. Urinary continence at 6 and 12 months after RARP was associated with age at the time of surgery $(P<0.05)$. Data from the 24 - and 48 -month follow-ups are presented in Table 4. Patients in the incontinence group were older than those in the continence group at 24-month follow-up $(P<0.05)$. No significant variables were observed at the 48-month follow-up.

Univariate and multivariate associations between urinary continence and predictive factors are shown in Tables 5, 6, and 7. In the univariate logistic analysis, PLND was associated with urinary continence at 1 -month post-surgery $(P=0.009)$. In the multivariate logistic analysis, PLND was a significant independent risk factor of early urinary continence at 1 month (odds ratio (OR): 1.535, 95\% confidence interval $(\mathrm{CI})$ : 1.079-2.184, $P=0.017$; Table 5).

Univariate logistic analysis showed that NS was associated with urinary continence at 1 month (OR: 0.372, 95\% CI: $0.248-0.557, P<0.001$ ), 3 months (OR: 0.549, 95\% CI: $0.356-0.846, P=0.007$ ), and 6 months (OR: 0.561, 95\% CI: 0.346-0.909, $P=0.019$ ) (Tables 5 and 6). In multivariate logistic analysis, NS was a significant independent protective factor of urinary continence at 1 month (OR: 0.360, 95\% CI: 0.231-0.561, $P<0.001$ ), 3 months (OR: $0.546,95 \% \mathrm{CI}: 0.342-0.872, P=0.011$ ), and 6 months (OR: 0.545, 95\% CI: 0.324-0.915, $P=0.022$ ) (Tables 5 and 6).

Univariate logistic analysis showed that younger age was associated with urinary continence at 6 months (OR: 1.028, 95\% CI: $1.005-1.052, P=0.019), 12$ months (OR: 1.034, 95\% CI: $1.007-1.061, P=0.012$ ), and 24 months (OR: 1.970, 95\% CI: 1.943-1.998, $P=0.017$ ) (Tables 6 and 7). In multivariate logistic analysis, advanced age 
Table 3 Comparison of parameters between continence and incontinence patients at 6 and 12 months after operation

\begin{tabular}{|c|c|c|c|c|c|c|}
\hline \multirow[t]{2}{*}{ Variable } & \multicolumn{3}{|l|}{6 Months } & \multicolumn{3}{|l|}{12 Months } \\
\hline & Continence $(N=464)$ & Incontinence $(N=186)$ & $P$ value & Continence $(N=512)$ & Incontinence $(N=138)$ & $P$ value \\
\hline Age (years) & $65.17 \pm 7.33$ & $66.73 \pm 8.08$ & 0.018 & $65.23 \pm 7.36$ & $67.06 \pm 8.22$ & 0.012 \\
\hline BMI $\left(\mathrm{kg} / \mathrm{m}^{2}\right)$ & $25.05 \pm 2.78$ & $24.93 \pm 3.14$ & 0.631 & $25.07 \pm 2.84$ & $24.82 \pm 3.06$ & 0.362 \\
\hline \multicolumn{7}{|l|}{ Comorbidities, no (\%) } \\
\hline $\mathrm{HP}$ & 150 (32.33\%) & $68(36.56 \%)$ & 0.302 & 172 (33.59\%) & $46(33.33 \%)$ & 0.954 \\
\hline $\mathrm{DM}$ & $73(15.73 \%)$ & $27(14.52 \%)$ & 0.698 & $80(15.63 \%)$ & $20(14.49 \%)$ & 0.744 \\
\hline $\mathrm{CHD}$ & $41(8.84 \%)$ & $22(11.83 \%)$ & 0.244 & $46(8.98 \%)$ & $17(12.32 \%)$ & 0.240 \\
\hline$C D$ & $12(2.59 \%)$ & $8(4.30 \%)$ & 0.253 & $13(2.54 \%)$ & 7 (5.07\%) & 0.126 \\
\hline PSA (ng/mL) & $29.15 \pm 33.12$ & $33.16 \pm 38.85$ & 0.185 & $29.43 \pm 32.66$ & $32.51 \pm 42.07$ & 0.223 \\
\hline PV $(m L)$ & $37.14 \pm 19.11$ & $35.20 \pm 19.63$ & 0.245 & $36.95 \pm 18.75$ & $35.24 \pm 21.10$ & 0.354 \\
\hline Gleason score, no (\%) & & & 0.251 & & & 0.257 \\
\hline$\leq 6$ & 137 (29.53\%) & $56(30.11 \%)$ & & 153 (29.88\%) & $40(28.99 \%)$ & \\
\hline 7 & 178 (38.36\%) & 77 (41.40\%) & & 197 (38.48\%) & $58(42.03 \%)$ & \\
\hline$\geq 8$ & 133 (28.66\%) & $42(22.58 \%)$ & & 144 (28.13\%) & $31(22.46 \%)$ & \\
\hline Unknown & $16(3.45 \%)$ & $11(5.91 \%)$ & & $18(3.51 \%)$ & $9(6.52 \%)$ & \\
\hline Clinical T stage, no (\%) & & & 0.659 & & & 0.918 \\
\hline $\mathrm{T} 1 \mathrm{a}, \mathrm{b}$ & $2(0.43 \%)$ & 0 & & $2(0.38 \%)$ & 0 & \\
\hline T1c & 78 (16.81\%) & $36(19.35 \%)$ & & $88(17.19 \%)$ & $26(18.84 \%)$ & \\
\hline $\mathrm{T} 2 \mathrm{a}, \mathrm{b}$ & 178 (38.36\%) & $63(33.87 \%)$ & & 193 (37.70\%) & $48(34.78 \%)$ & \\
\hline $\mathrm{T} 2 \mathrm{C}$ & 179 (38.58\%) & 79 (42.47\%) & & 201 (39.26\%) & $57(41.30 \%)$ & \\
\hline ТЗа & $2(0.43 \%)$ & $1(0.55 \%)$ & & $2(0.38 \%)$ & $1(0.73 \%)$ & \\
\hline T3b & 25 (5.39\%) & 7 (3.76\%) & & $26(5.08 \%)$ & $6(4.35 \%)$ & \\
\hline OT (mins) & $152.42 \pm 44.08$ & $154.15 \pm 51.17$ & 0.666 & $151.66 \pm 43.98$ & $157.57 \pm 53.49$ & 0.182 \\
\hline NS, no (\%) & 97 (20.91\%) & $24(12.90 \%)$ & 0.018 & 103 (20.12\%) & $18(13.04 \%)$ & 0.058 \\
\hline PLND, no (\%) & 241 (51.94\%) & 97 (52.15\%) & 0.961 & 267 (52.15\%) & 71 (51.45\%) & 0.884 \\
\hline DIC (days) & $21.34 \pm 11.20$ & $20.51 \pm 8.00$ & 0.356 & $21.37 \pm 10.96$ & $20.13 \pm 7.83$ & 0.215 \\
\hline
\end{tabular}

Abbreviations: BMI Body mass index, CHD Coronary heart disease, CD Cerebrovascular diseases, DM Diabetes mellitus, DIC Duration of indwelling catheter, HP Hypertension, NS Nerve-sparing, OT Operation time, PSA Prostate-specific antigen, PV Prostate volume, PLND Pelvic lymph node dissection

was a significant independent risk factor of urinary continence at 6 months (OR: 1.026, 95\% CI: 1.001-1.051, $P=0.038$ ), 12 months (OR: 1.030, 95\% CI: 1.002-1.058, $P=0.035$ ), and 24 months (OR: 1.968, 95\% CI: 1.9391.997, $P=0.038$ ) (Tables 6 and 7). Other variables mentioned above were not statistically significant predictors. Also, no significant predictors of late urinary continence were detected at 48 months (Table 7).

\section{Discussion}

Reportedly, the urinary continence is stable up to 12 months after RP [2]. Hence, previous studies have addressed CRs within 1 year post-RARP. Only little data are available at more than 24 months follow-up for urinary continence. In the current study, the CRs were $78.77,79.96,79.51$, and $76.50 \%$ at $1,2,3$, and 4 years after the surgery, respectively (Table 1). No significant differences were observed in the continence outcomes during the 4-year follow-up. Our results certified that one year after RARP was the stable continence period [15]. Few studies have evaluated the CRs after 12 months. Shao et al. reported that CR was $89.4 \%$ at 24 months after RARP, while Xylinas et al. reported that the 24 -month urinary continence rate was $88 \%$ based on the no-pad definition [7, 9]. Murphy et al. reported a 36month urinary continence rate of $94.7 \%$ using the nopad or safety pad definition [10]. Mandel et al. reported that CRs were 89.5 and $90.9 \%$ at 24 and 36 months after the surgery, respectively [16]. The CR of the current study was $76.50 \%$ at 48 months after RARP, which has been the longest follow-up on the topic to date.

A large number of studies have evaluated the predictors of urinary continence within 1 year after surgery. These studies either included a relatively small number of patients or had a discontinuous follow-up. To the best of our knowledge, this is the first study to evaluate the predictors of continence from 1 to 48 months after RARP in a large sample. 
Table 4 Comparison of parameters between continence and incontinence patients at 24 and 48 months after operation

\begin{tabular}{|c|c|c|c|c|c|c|}
\hline \multirow[t]{2}{*}{ Variable } & \multicolumn{3}{|l|}{24 Months } & \multicolumn{3}{|l|}{48 Months } \\
\hline & Continence $(N=375)$ & Incontinence $(N=94)$ & $P$ value & Continence $(N=166)$ & Incontinence $(N=51)$ & $P$ value \\
\hline Age (years) & $63.93 \pm 8.48$ & $65.82 \pm 7.62$ & 0.016 & $65.70 \pm 7.78$ & $66.16 \pm 7.97$ & 0.719 \\
\hline BMI $\left(\mathrm{kg} / \mathrm{m}^{2}\right)$ & $25.16 \pm 2.91$ & $24.71 \pm 3.05$ & 0.186 & $25.21 \pm 2.89$ & $25.30 \pm 2.61$ & 0.832 \\
\hline \multicolumn{7}{|l|}{ Comorbidities, no (\%) } \\
\hline $\mathrm{HP}$ & $138(36.80 \%)$ & 27 (28.72\%) & 0.143 & 53 (31.93\%) & 20 (39.22\%) & 0.335 \\
\hline DM & $60(16.00 \%)$ & $11(11.70 \%)$ & 0.299 & $25(15.06 \%)$ & 13 (25.49\%) & 0.087 \\
\hline $\mathrm{CHD}$ & $40(10.67 \%)$ & $6(6.38 \%)$ & 0.212 & $21(12.65 \%)$ & $6(11.76 \%)$ & 0.867 \\
\hline$C D$ & $11(2.93 \%)$ & $4(4.26 \%)$ & 0.515 & $5(3.01 \%)$ & $2(3.92 \%)$ & 0.748 \\
\hline PSA (ng/mL) & $30.00 \pm 34.03$ & $33.77 \pm 35.59$ & 0.343 & $30.57 \pm 40.59$ & $35.60 \pm 38.12$ & 0.433 \\
\hline PV (mL) & $35.97 \pm 19.66$ & $34.72 \pm 15.95$ & 0.567 & $34.50 \pm 18.85$ & $40.11 \pm 20.82$ & 0.071 \\
\hline Gleason score, no (\%) & & & 0.336 & & & 0.501 \\
\hline$\leq 6$ & 109 (29.07\%) & 29 (30.85\%) & & 49 (29.52\%) & 20 (39.22\%) & \\
\hline 7 & $151(40.27 \%)$ & $42(44.68 \%)$ & & $63(37.95 \%)$ & 19 (37.25\%) & \\
\hline$\geq 8$ & $103(27.47 \%)$ & 18 (19.15\%) & & 47 (28.31\%) & $11(21.57 \%)$ & \\
\hline Unknown & $12(3.19 \%)$ & $5(5.32 \%)$ & & 7 (4.22\%) & $1(1.96 \%)$ & \\
\hline Clinical T stage, no (\%) & & & 0.806 & & & 0.356 \\
\hline $\mathrm{T} 1 \mathrm{a}, \mathrm{b}$ & $1(0.27 \%)$ & 0 & & 0 & 0 & \\
\hline T1c & $68(18.13 \%)$ & 15 (15.96\%) & & 32 (19.28\%) & 8 (15.69\%) & \\
\hline $\mathrm{T} 2 \mathrm{a}, \mathrm{b}$ & 133 (35.47\%) & 39 (41.49\%) & & 54 (32.53\%) & $11(21.57 \%)$ & \\
\hline $\mathrm{T} 2 \mathrm{C}$ & $152(40.53 \%)$ & $34(36.17 \%)$ & & 70 (42.17\%) & $29(56.86 \%)$ & \\
\hline Т3а & $2(0.53 \%)$ & 0 & & $2(1.20 \%)$ & 0 & \\
\hline $\mathrm{T} 3 \mathrm{~b}$ & $19(5.07 \%)$ & $6(6.38 \%)$ & & 8 (4.82\%) & $3(5.88 \%)$ & \\
\hline OT (mins) & $155.19 \pm 48.98$ & $146.79 \pm 42.32$ & 0.128 & $154.39 \pm 49.88$ & $152.14 \pm 53.29$ & 0.782 \\
\hline NS, no (\%) & 65 (17.33\%) & $12(12.77 \%)$ & 0.285 & 20 (12.05\%) & 8 (15.69\%) & 0.498 \\
\hline PLND, no (\%) & 204 (54.40\%) & $52(55.32 \%)$ & 0.873 & $91(54.82 \%)$ & 33 (64.71\%) & 0.212 \\
\hline DIC (days) & $21.27 \pm 11.21$ & $19.94 \pm 5.08$ & 0.260 & $21.14 \pm 12.23$ & $20.20 \pm 7.93$ & 0.603 \\
\hline
\end{tabular}

Abbreviations: BMI Body mass index, CHD Coronary heart disease, $C D$ Cerebrovascular diseases, DM Diabetes mellitus, DIC Duration of indwelling catheter, HP Hypertension, NS Nerve-sparing, OT Operation time, PSA Prostate-specific antigen, PV Prostate volume, PLND Pelvic lymph node dissection

In the current cohort, the CRs were 40.62, 60.92, 71.38 , and $78.77 \%$ at $1,3,6$ and 12 months after RARP, respectively (Table 1 ). These results were in agreement with those from the recent study by Honda et al. that revealed CRs at 1,3 , and 6 months as 40.7, 63.0, and $73.1 \%$, respectively [13]. The definition of postoperative urinary continence varied among several studies. Hitherto, there is no consensus on UI post-RP [17]. Herein, we selected the most stringent definition of incontinence: any leakage of urine. Reportedly, the continence rate one year after RARP is $69-97 \%[1,18]$. The overall continence rate in this study was $78.77 \%$ at 12 months, without the usage of any pad. Although it is not excellent, it is within the average range.

To identify the predictive factors, urinary continence was divided into two categories: early continence ( $<3$ months) and late continence ( $>12$ months) [12].

In general, PLND was selectively performed for sampling purposes in intermediate- and high-risk patients
[14]. One month after the surgery, PLND in the continence group occurred in 121 patients (45.83\%), while it occurred in 217 patients in the incontinence group $(56.22 \%)(P<0.05)$. Three months post-surgery, no significant differences were detected in both groups. The logistic analysis showed that PLND was a significant independent risk factor of early urinary continence at 1 month. Patients who had undergone PLND during surgery had a high risk of UI. Lymphadenectomy may give rise to transient damage to nerve vessel bundles (NVBs), which affected the recovery of urinary continence. However, with the recovery of body function, this impact declined gradually.

The current logistic analysis showed that NS was a significant independent protective factor of urinary continence at 1,3 , and 6 months (Tables 5 and 6 ). These findings were in line with those from the study by Reeves et al., which found that avoiding damage to the nerves around the prostate improves urinary continence in the first 6 months after 
Table 5 Univariable and multivariable regression analysis for predictors of continence 1 and 3 months

\begin{tabular}{|c|c|c|c|c|c|c|c|c|}
\hline \multirow[t]{3}{*}{ Predictors } & \multicolumn{4}{|l|}{1 Month } & \multicolumn{4}{|l|}{3 Months } \\
\hline & \multicolumn{2}{|l|}{ Univariable } & \multicolumn{2}{|l|}{ Multivariable } & \multicolumn{2}{|l|}{ Univariable } & \multicolumn{2}{|l|}{ Multivariable } \\
\hline & $\mathrm{OR}, 95 \% \mathrm{Cl}$ & $P$ value & $\mathrm{OR}, 95 \% \mathrm{Cl}$ & $P$ value & OR, 95\% Cl & $P$ value & $\mathrm{OR}, 95 \% \mathrm{Cl}$ & $P$ value \\
\hline Age & $1.001,0.980-1.021$ & 0.959 & $1.001,0.979-1.023$ & 0.940 & $1.019,0.998-1.041$ & 0.080 & $1.018,0.996-1.041$ & 0.113 \\
\hline BMl & $1.036,0.981-1.094$ & 0.209 & $1.023,0.963-1.086$ & 0.460 & $1.001,0.948-1.057$ & 0.964 & $0.998,0.941-1.059$ & 0.947 \\
\hline $\mathrm{HP}$ & $1.398,0.998-1.957$ & 0.051 & $1.376,0.947-1.999$ & 0.094 & $1.365,0.980-1.901$ & 0.066 & $1.404,0.978-2.015$ & 0.066 \\
\hline DM & $1.138,0.734-1.764$ & 0.563 & $0.999,0.626-1.595$ & 0.998 & $0.857,0.550-1.334$ & 0.493 & $0.748,0.470-1.190$ & 0.220 \\
\hline CHD & $1.529,0.878-2.665$ & 0.134 & $1.476,0.817-2.688$ & 0.197 & $1.028,0.605-1.750$ & 0.917 & $0.885,0.508-1.542$ & 0.665 \\
\hline$C D$ & $0.831,0.340-2.034$ & 0.685 & $0.739,0.289-1.889$ & 0.528 & $1.582,0.649-3.856$ & 0.313 & $1.456,0.582-3.640$ & 0.422 \\
\hline PSA & $1.004,0.999-1.009$ & 0.114 & $1.000,0.995-1.005$ & 0.985 & $1.003,0.999-1.008$ & 0.138 & $1.002,0.997-1.006$ & 0.501 \\
\hline PV & $0.995,0.987-1.003$ & 0.212 & $0.994,0.986-1.002$ & 0.159 & $0.996,0.988-1.004$ & 0.348 & $0.995,0.987-1.004$ & 0.262 \\
\hline Gleason score & $0.929,0.774-1.116$ & 0.433 & $0.801,0.654-0.981$ & 0.332 & $0.965,0.802-1.160$ & 0.702 & $0.926,0.758-1.131$ & 0.449 \\
\hline Clinical stage & $1.060,0.899-1.250$ & 0.487 & $1.013,0.850-1.208$ & 0.883 & $1.077,0.913-1.270$ & 0.379 & $1.071,0.902-1.273$ & 0.434 \\
\hline OT & $1.001,0.998-1.004$ & 0.551 & $1.000,0.996-1.003$ & 0.931 & $1.002,0.999-1.006$ & 0.193 & $1.002,0.998-1.005$ & 0.281 \\
\hline NS & $0.372,0.248-0.557$ & 0.000 & $0.360,0.231-0.561$ & 0.000 & $0.549,0.356-0.846$ & 0.007 & $0.546,0.342-0.872$ & 0.011 \\
\hline PLND & $1.517,1.018-2.079$ & 0.009 & $1.535,1.079-2.184$ & 0.017 & $1.051,0.767-1.441$ & 0.757 & $0.978,0.690-1.386$ & 0.901 \\
\hline $\mathrm{DIC}$ & $0.998,0.983-1.013$ & 0.809 & $0.999,0.983-1.014$ & 0.869 & $0.996,0.980-1.012$ & 0.602 & $0.997,0.981-1.013$ & 0.713 \\
\hline
\end{tabular}

Abbreviations: BMI Body mass index, CHD Coronary heart disease, CD Cerebrovascular diseases, DM Diabetes mellitus, DIC Duration of indwelling catheter, HP Hypertension, NS Nerve-sparing, OT Operation time, PSA Prostate-specific antigen, PV Prostate volume, PLND Pelvic lymph node dissection

the surgery [19]. Michl et al. investigated long-term CRs (12 months) after RP and found a significant difference between the NS and non-NS groups [20]. The studies by Kadono et al. and Steineck et al. also indicated that NS is associated with urinary continence in the long-term $[15,21]$. The bias in the studies used for the analysis might have influenced the results. However, all the studies showed that NS during the surgery produced satisfactory postoperative continence outcomes.

Multiple studies have demonstrated that age is an independent risk factor for the return of continence at 112 months after RARP. Lavigueur-Blouin et al. evaluated the early continence after RARP [22]. It showed that advanced age was an independent predictor at 1 month. Kim et al. demonstrated that younger men could have

Table 6 Univariable and multivariable regression analysis for predictors of continence 6 and 12 months

\begin{tabular}{|c|c|c|c|c|c|c|c|c|}
\hline \multirow[t]{3}{*}{ Predictors } & \multicolumn{4}{|l|}{6 Months } & \multicolumn{4}{|l|}{12 Months } \\
\hline & \multicolumn{2}{|l|}{ Univariable } & \multicolumn{2}{|l|}{ Multivariable } & \multicolumn{2}{|l|}{ Univariable } & \multicolumn{2}{|l|}{ Multivariable } \\
\hline & $\mathrm{OR}, 95 \% \mathrm{Cl}$ & $P$ value & $\mathrm{OR}, 95 \% \mathrm{Cl}$ & $P$ value & $\mathrm{OR}, 95 \% \mathrm{Cl}$ & $P$ value & $\mathrm{OR}, 95 \% \mathrm{Cl}$ & $P$ value \\
\hline Age & $1.028,1.005-1.052$ & 0.019 & $1.026,1.001-1.051$ & 0.038 & $1.034,1.007-1.061$ & 0.012 & $1.030,1.002-1.058$ & 0.035 \\
\hline BMl & $0.986,0.929-1.046$ & 0.630 & $0.993,0.932-1.058$ & 0.827 & $0.970,0.908-1.036$ & 0.362 & $0.983,0.916-1.054$ & 0.626 \\
\hline $\mathrm{HP}$ & $1.206,0.845-1.723$ & 0.302 & $1.179,0.800-1.737$ & 0.404 & $0.988,0.663-1.472$ & 0.954 & $0.937,0.606-1.448$ & 0.768 \\
\hline DM & $0.910,0.564-1.468$ & 0.698 & $0.834,0.506-1.375$ & 0.477 & $0.915,0.538-1.556$ & 0.744 & $0.873,0.501-1.521$ & 0.632 \\
\hline CHD & $1.384,0.800-2.395$ & 0.246 & $1.190,0.672-2.109$ & 0.551 & $1.423,0.788-2.571$ & 0.242 & $1.275,0.686-2.371$ & 0.443 \\
\hline$C D$ & $1.693,0.681-4.211$ & 0.258 & $1.583,0.617-4.064$ & 0.339 & $2.051,0.802-5.244$ & 0.134 & $2.144,0.806-5.072$ & 0.126 \\
\hline PSA & $1.003,0.998-1.008$ & 0.190 & $1.001,0.996-1.006$ & 0.614 & $1.003,0.998-1.008$ & 0.227 & $1.002,0.996-1.007$ & 0.517 \\
\hline PV & $0.995,0.985-1.004$ & 0.245 & $0.995,0.985-1.004$ & 0.288 & $0.995,0.985-1.005$ & 0.354 & $0.995,0.985-1.006$ & 0.393 \\
\hline Gleason score & $0.976,0.800-1.192$ & 0.815 & $0.946,0.764-1.173$ & 0.614 & $1.017,0.816-1.267$ & 0.879 & $0.998,0.788-1.264$ & 0.989 \\
\hline Clinical stage & $0.973,0.814-1.164$ & 0.767 & $0.972,0.807-1.171$ & 0.765 & $0.996,0.818-1.214$ & 0.970 & $0.991,0.807-1.218$ & 0.932 \\
\hline OT & $1.001,0.997-1.004$ & 0.665 & $1.001,0.997-1.005$ & 0.720 & $1.003,0.999-1.007$ & 0.182 & $1.003,0.999-1.007$ & 0.177 \\
\hline NS & $0.561,0.346-0.909$ & 0.019 & $0.545,0.324-0.915$ & 0.022 & $0.596,0.347-1.023$ & 0.060 & $0.609,0.342-1.086$ & 0.093 \\
\hline PLND & $1.008,0.717-1.418$ & 0.961 & $0.993,0.683-1.444$ & 0.971 & $0.972,0.668-1.416$ & 0.884 & $0.914,0.606-1.380$ & 0.670 \\
\hline DIC & $0.991,0.973-1.010$ & 0.359 & $0.993,0.975-1.012$ & 0.474 & $0.986,0.964-1.008$ & 0.215 & $0.988,0.966-1.011$ & 0.304 \\
\hline
\end{tabular}

Abbreviations: BMI Body mass index, CHD Coronary heart disease, CD Cerebrovascular diseases, DM Diabetes mellitus, DIC Duration of indwelling catheter, HP Hypertension, NS Nerve-sparing, OT Operation time, PSA Prostate-specific antigen, PV Prostate volume, PLND Pelvic lymph node dissection 
Table 7 Univariable and multivariable regression analysis for predictors of continence 24 and 48 months

\begin{tabular}{|c|c|c|c|c|c|c|c|c|}
\hline \multirow[t]{3}{*}{ Predictors } & \multicolumn{4}{|l|}{24 Months } & \multicolumn{4}{|l|}{48 Months } \\
\hline & \multicolumn{2}{|l|}{ Univariable } & \multicolumn{2}{|l|}{ Multivariable } & \multicolumn{2}{|l|}{ Univariable } & \multicolumn{2}{|l|}{ Multivariable } \\
\hline & $\mathrm{OR}, 95 \% \mathrm{Cl}$ & $P$ value & $\mathrm{OR}, 95 \% \mathrm{Cl}$ & $P$ value & OR, 95\% Cl & $P$ value & $\mathrm{OR}, 95 \% \mathrm{Cl}$ & $P$ value \\
\hline Age & $1.970,1.943-1.998$ & 0.017 & $1.968,1.939-1.997$ & 0.038 & $1.008,0.967-1.049$ & 0.717 & $1.009,0.964-1.057$ & 0.691 \\
\hline BMl & $0.949,0.877-1.026$ & 0.186 & $0.956,0.877-1.042$ & 0.310 & $1.012,0.906-1.131$ & 0.831 & $0.999,0.880-1.136$ & 0.992 \\
\hline $\mathrm{HP}$ & $0.692,0.422-1.134$ & 0.144 & $0.810,0.474-1.384$ & 0.441 & $1.376,0.718-2.635$ & 0.336 & $1.484,0.708-3.109$ & 0.296 \\
\hline DM & $0.696,0.350-1.383$ & 0.301 & $0.779,0.382-1.589$ & 0.492 & $1.929,0.902-4.125$ & 0.090 & $1.803,0.803-4.051$ & 0.153 \\
\hline CHD & $0.571,0.235-1.390$ & 0.217 & $0.642,0.255-1.617$ & 0.347 & $0.921,0.350-2.421$ & 0.867 & $0.847,0.293-2.448$ & 0.759 \\
\hline$C D$ & $1.471,0.458-4.726$ & 0.517 & $1.816,0.535-6.160$ & 0.339 & $1.314,0.247-6.987$ & 0.749 & $1.003,0.158-6.389$ & 0.997 \\
\hline PSA & $1.003,0.997-1.009$ & 0.346 & $1.003,0.996-1.009$ & 0.419 & $1.003,0.996-1.010$ & 0.437 & $1.002,0.995-1.010$ & 0.530 \\
\hline PV & $0.996,0.984-1.009$ & 0.567 & $0.996,0.983-1.009$ & 0.580 & $1.014,0.999-1.029$ & 0.077 & $1.014,0.998-1.031$ & 0.093 \\
\hline Gleason score & $0.919,0.699-1.207$ & 0.542 & $0.892,0.659-1.208$ & 0.461 & $0.744,0.509-1.089$ & 0.128 & $0.691,0.452-1.058$ & 0.089 \\
\hline Clinical stage & $1.013,0.802-1.280$ & 0.912 & $1.054,0.824-1.348$ & 0.677 & $1.222,0.886-1.684$ & 0.221 & $1.189,0.835-1.692$ & 0.337 \\
\hline OT & $0.996,0.991-1.001$ & 0.128 & $0.997,0.991-1.002$ & 0.210 & $0.999,0.993-1.005$ & 0.781 & $0.998,0.991-1.005$ & 0.492 \\
\hline NS & $0.698,0.360-1.353$ & 0.287 & $0.719,0.352-1.472$ & 0.367 & $1.358,0.559-3.299$ & 0.499 & $1.163,0.427-3.167$ & 0.767 \\
\hline PLND & $1.038,0.659-1.635$ & 0.873 & $1.145,0.690-1.902$ & 0.600 & $1.511,0.788-2.896$ & 0.214 & $1.720,0.828-3.573$ & 0.146 \\
\hline $\mathrm{DIC}$ & $0.983,0.955-1.012$ & 0.257 & $0.980,0.949-1.013$ & 0.228 & $0.991,0.958-1.026$ & 0.605 & $0.989,0.950-1.030$ & 0.604 \\
\hline
\end{tabular}

Abbreviations: BMI Body mass index, CHD Coronary heart disease, CD Cerebrovascular diseases, DM Diabetes mellitus, DIC Duration of indwelling catheter, HP Hypertension, NS Nerve-sparing, OT Operation time, PSA Prostate-specific antigen, PV Prostate volume, PLND Pelvic lymph node dissection

an early recurrence of continence 3 months after RARP [23]. Greco et al. compared the continence outcomes of RARP in older men to those of the younger men [24]. The study showed that CRs at 1,3 , and 12 months were similar in the two groups; however, the older group had a significantly lower continence rate at 6 months. The results of these studies might partially be in agreement with those of our study. Shikanov et al. demonstrated that age is a predictor of continence return at 12 months after RARP, which is partially in accordance with the current results [25]. Our results show that advanced age is a significant risk factor of continence at 6,12 , and 24 months after the surgery (Tables 6 and 7). Men of advanced age had a high risk of UI. Older men have poor endothelial dysfunction, which affects the vascular supply of the NVBs. In addition, it is difficult to perform pelvic floor exercises (PLE) due to an age-related decrease in the mass of the skeletal muscle and neuronal plasticity [26]. These conditions might affect functional outcomes.

Interestingly, the optimal time to remove the indwelling catheter has not yet been determined. Conventionally, the urinary catheter was removed between 10 and 21 days postoperatively [27]. Typically, the catheter is removed 3 weeks after the operation in our center in order to ensure the healing of anastomosis. However, most patients returned home after the operation, and the indwelling catheter was removed at the local clinic. This leads to the inconsistency of catheter removal and the duration of indwelling catheter might be longer than expected.

\section{Limitations}

Nevertheless, the present study had some limitations. First, this was a retrospective study from a single institution, and surgeries were not performed by a single surgeon. Second, we did not analyze all variables due to the undocumented surgical steps of the procedure, variations in surgical experience, and differences in the pathological reports. Third, although a stringent definition of continence was applied, the conditions were reported by the patients rather than based on a quality questionnaire. Fourth, the potential bias in selecting the patients for the procedure might influence the results. In addition, data were missing as many patients were lost to follow-up. Despite these drawbacks, this study utilized a large sample size, and the survey of postoperative urinary continence was time-continuous.

\section{Conclusions}

The current study has shown that CR improves gradually with time within 1 year post-surgery and stabilizes after one more year. PLND, NS, and age are the significant determinants of continence in the early and late stages, respectively. Thus, these parameters could be used to identify patients at high risk for UI preoperatively and counsel them on postoperative expectations for urinary continence.

\section{Abbreviations}

BMI: Body mass index; CRs: Continence rates; CHD: Coronary heart disease; CD: Cerebrovascular diseases; Cl: Confidence interval; DM: Diabetes mellitus; DIC: Duration of indwelling catheter; HP: Hypertension; LRP: Laparoscopic radical prostatectomy; NS: Nerve-sparing; NVBs: Nerve vessel bundles; 
OT: Operation time; OR: Odds ratio; RARP: Robot-assisted radical prostatectomy; RRP: Retropubic radical prostatectomy; PSA: Prostate-specific antigen; PV: Prostate volume; PLND: Pelvic lymph node dissection; PLE: Pelvic floor exercises; RP: Radical prostatectomy; UI: Urinary incontinence

\section{Acknowledgements}

Not applicable.

\section{Authors' contributions}

XL: Protocol/project development, Data Collection, Data management, Statistical analysis, Manuscript writing HZ: Data Collection, Data analysis ZJ: Data Collection, Data analysis YW: Data Collection, Data analysis YS: Data Collection, Data analysis LL: Critical revision of the manuscript, Manuscript editing XZ: Critical revision of the manuscript, Manuscript editing. All authors read and approved the final version of the manuscript.

\section{Funding}

This study received financial support from China Postdoctoral Science Foundation funded project (2018 M643880) and National Natural Science Foundation of China (81570688).

\section{Availability of data and materials}

All the data supporting our findings is contained in the manuscript. The datasets used and/or analysed in the current study is available from the corresponding author on reasonable request.

\section{Ethics approval and consent to participate}

All procedures performed in studies involving human participants were in accordance with the ethical standards of the institutional and/or national research committee and with the 1964 Helsinki declaration and its later amendments or comparable ethical standards. Written informed consent was obtained from all individual participants included in the study. This study was approved by the Chinese PLA General Hospital Local Ethics Committee.

\section{Consent for publication}

Not applicable.

\section{Competing interests}

The authors declare that they have no competing interest.

Received: 16 October 2019 Accepted: 10 March 2020

Published online: 18 March 2020

\section{References}

1. Ficarra V, Novara G, Rosen RC, Artibani W, Carroll PR, Costello A, Menon M, Montorsi F, Patel VR, Stolzenburg JU, Van der Poel H, Wilson TG, Zattoni F, Mottrie A. Systematic review and meta-analysis of studies reporting urinary continence recovery after robot-assisted radical prostatectomy. Eur Urol. 2012;62(3):405-17.

2. Litwin MS, Pasta DJ, Yu J, Stoddard ML, Flanders SC. Urinary function and bother after radical prostatectomy or radiation for prostate cancer: a longitudinal, multivariate quality of life analysis from the Cancer of the prostate strategic urologic research endeavor. J Urol. 2000;164(6):1973-7.

3. Bauer RM, Gozzi C, Hübner W, Nitti WW, Novara G, Peterson A, Sandhu JS, Stief CG. Contemporary management of postprostatectomy incontinence. Eur Urol. 2011;59(6):985-96

4. Suardi N, Moschini M, Gallina A, Gandaglia G, Abdollah F, Capitanio U, Bianchi M, Tutolo M, Passoni N, Salonia A, Hedlund P, Rigatti P, Montorsi F, Briganti A. Nerve-sparing approach during radical prostatectomy is strongly associated with the rate of postoperative urinary continence recovery. BJU Int. 2013;111(5):717-22.

5. Sato Y, Tanda H, Nakajima H, Nitta T, Akagashi K, Hanzawa T, Tobe M, Haga K, Uchida K, Honma I. Simple and reliable predictor of urinary continence after radical prostatectomy: serial measurement of urine loss ratio after catheter removal. Int J Urol. 2014;21(7):647-51.

6. Nakagawa $T$, Kadono $Y$, Naito $R$, Iwamoto $H$, Yaegashi H, lijima M, Kawaguchi S, Nohara T, Shigehara K, Izumi K, Mizokami A. Factors associated with treatment satisfaction after robot-assisted radical prostatectomy. Anticancer Res. 2019;39(11):6339-46.

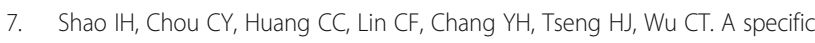
cystography pattern can predict postprostatectomy incontinence. Ann Surg Oncol. 2015;22(Suppl 3):1580-6.

8. Haga N, Aikawa K, Hoshi S, Yabe M, Akaihata H, Hata J, Satoh Y, Ogawa S, Ishibashi K, Kojima Y. Postoperative urinary incontinence exacerbates nocturia-specific quality of life after robot-assisted radical prostatectomy. Int J Urol. 2016;23(10):873-8.

9. Xylinas E, Durand X, Ploussard G, Campeggi A, Allory Y, Vordos D, Hoznek A, Abbou CC, de la Taille A, Salomon L. Evaluation of combined oncologic and functional outcomes after robotic-assisted laparoscopic extraperitoneal radical prostatectomy: trifecta rate of achieving continence, potency and cancer control. Urol Oncol. 2013;31(1):99-103.

10. Murphy DG, Kerger M, Crowe H, Peters JS, Costello AJ. Operative details and oncological and functional outcome of robotic-assisted laparoscopic radical prostatectomy: 400 cases with a minimum of 12 months follow-up. Eur Urol. 2009;55(6):1358-66.

11. Yanagiuchi A, Miyake H, Tanaka K, Fujisawa M. Significance of preoperatively observed detrusor overactivity as a predictor of continence status early after robot-assisted radical prostatectomy. Asian J Androl. 2014;16(6):869-72.

12. Olgin G, Alsyouf M, Han D, Li R, Lightfoot M, Smith D, Nicolay L, Ruckle H, Baldwin DD. Postoperative cystogram findings predict incontinence following robot-assisted radical prostatectomy. J Endourol. 2014;28(12): 1460-3.

13. Honda M, Kawamoto B, Morizane S, Hikita K, Muraoka K, Sejima T, Takenaka A. A prognostic model for predicting urinary incontinence after robotassisted radical prostatectomy. Int J Med Robot. 2017;13(3):1-8.

14. Heidenreich A, Bellmunt J, Bolla M, Joniau S, Mason M, Matveev V, Mottet N Schmid HP, van der Kwast T, Wiegel T. Zattoni F; European Association of Urology. EAU guidelines on prostate cancer. Part 1: screening, diagnosis, and treatment of clinically localised disease. Eur Urol. 2011;59(1):61-71.

15. Kadono $Y$, Ueno S, Kadomoto S, Iwamoto H, Takezawa Y, Nakashima K, Nohara T, Izumi K, Mizokami A, Gabata T, Namiki M. Use of preoperative factors including urodynamic evaluations and nerve-sparing status for predicting urinary continence recovery after robot-assisted radical prostatectomy: nerve-sparing technique contributes to the reduction of postprostatectomy incontinence. Neurourol Urodyn. 2016;35(8):1034-9.

16. Mandel P, Preisser F, Graefen M, Steuber T, Salomon G, Haese A, Michl U, Huland $\mathrm{H}$, Tilki D. High chance of late recovery of urinary and erectile function beyond 12 months after radical prostatectomy. Eur Urol. 2017; 71(6):848-50.

17. Cestari A, Rigatti L, Lughezzani G, Guazzoni G. How should we report incontinence after radical prostatectomy? J Urol. 2010;184(3):829-30.

18. Ficarra V, Novara G, Artibani W, Cestari A, Galfano A, Graefen M, Guazzoni G Guillonneau B, Menon M, Montorsi F, Patel V, Rassweiler J, Van Poppel H. Retropubic, laparoscopic, and robot-assisted radical prostatectomy: a systematic review and cumulative analysis of comparative studies. Eur Urol. 2009;55(5):1037-63.

19. Reeves F, Preece P, Kapoor J, Everaerts W, Murphy DG, Corcoran NM, Costello AJ. Preservation of the neurovascular bundles is associated with improved time to continence after radical prostatectomy but not long-term continence rates: results of a systematic review and meta-analysis. Eur Urol. 2015;68(4):692-704.

20. Michl U, Tennstedt P, Feldmeier L, Mandel P, Oh SJ, Ahyai S, Budäus L, Chun FKH, Haese A, Heinzer $H$, Salomon G, Schlomm T, Steuber T, Huland $H$, Graefen M, Tilki D. Nerve-sparing surgery technique, not the preservation of the neurovascular bundles, leads to improved long-term continence rates after radical prostatectomy. Eur Urol. 2016;69(4):584-9.

21. Steineck G, Bjartell A, Hugosson J, Axén E, Carlsson S, Stranne J, Wallerstedt A, Persson J, Wilderäng U, Thorsteinsdottir T, Gustafsson O, Lagerkvist M, Jiborn T, Haglind E, Wiklund P. LAPPRO steering committee. Degree of preservation of the neurovascular bundles during radical prostatectomy and urinary continence 1 year after surgery. Eur Urol. 2015;67(3):559-68.

22. Lavigueur-Blouin $H$, Noriega AC, Valdivieso R, Hueber PA, Bienz M, Alhathal $\mathrm{N}$, Latour M, Trinh QD, El-Hakim A, Zorn KC. Predictors of early continence following robot-assisted radical prostatectomy. Can Urol Assoc J. 2015;9(12):e93-7.

23. Kim JJ, Ha YS, Kim JH, Jeon SS, Lee DH, Kim WJ, Kim IY. Independent predictors of recovery of continence 3 months after robot-assisted laparoscopic radical prostatectomy. J Endourol. 2012;26(10):1290-5.

24. Greco KA, Meeks JJ, Wu S, Nadler RB. Robot-assisted radical prostatectomy in men aged > or $=70$ years. BJU Int. 2009;104(10):1492-5. 
25. Shikanov S, Desai V, Razmaria A, Zagaja GP, Shalhav AL. Robotic radical prostatectomy for elderly patients: probability of achieving continence and potency 1 year after surgery. J Urol. 2010;183(5):1803-7.

26. Kumar A, Samavedi S, Bates AS, Coelho RF, Rocco B, Palmer K, Patel VR. Continence outcomes of robot-assisted radical prostatectomy in patients with adverse urinary continence risk factors. BJU Int. 2015;116(5):764-70.

27. Matsushima M, Miyajima A, Hattori S, Takeda T, Mizuno R, Kikuchi E, Oya M. Comparison of continence outcomes of early catheter removal on postoperative day 2 and 4 after laparoscopic radical prostatectomy: a randomized controlled trial. BMC Urol. 2015;15:77.

\section{Publisher's Note}

Springer Nature remains neutral with regard to jurisdictional claims in published maps and institutional affiliations.

Ready to submit your research? Choose BMC and benefit from:

- fast, convenient online submission

- thorough peer review by experienced researchers in your field

- rapid publication on acceptance

- support for research data, including large and complex data types

- gold Open Access which fosters wider collaboration and increased citations

- maximum visibility for your research: over $100 \mathrm{M}$ website views per year

At $\mathrm{BMC}$, research is always in progress.

Learn more biomedcentral.com/submissions 